\title{
An Effective Statistics Approach to Secure Data File through Forbidden Zone Data Hiding Technique
}

\author{
Tyler Taylor, Justin Rogers
}

\begin{abstract}
Video Steganography is a strategy wherein we can conceal a wide range of documents with any extension into a carrying Video document. Right now, are utilizing two fundamental wording that is have host file and carrier file where host file is a hidden file (any sort of record like content document, picture document, and sound/video document) and carrier file must be a video record. The primary inspiration of this paper is to make sure about moving of information by utilizing steganography and cryptography system. It is worried about implanting data in a harmless spread media in a safe and hearty way. Right now expositions we are utilizing Forbidden Zone Data Hiding strategy where no modification is required in have signal range during information hidden procedure. To safely moving the information record, we use video information covering up and utilizing revision limit of rehash gather code with predominance of taboo zone information stowing away. Utilizing this methodology we can likewise stow away and move the enormous video record whose size is bigger than spread record in secure way. The principle favorable position of utilizing video record sequestered from everything data is the additional protection from of the outsider or unintended beneficiary because of the overall multifaceted nature of video contrasted with picture and sound document. I have effectively actualized the proposed system of video information hiding utilizing forbidden zone datahiding strategy (FZDH) on content document, picture record, sound record and video document. The exceptional element is that we can hide the bigger size video record behind the smaller size cover record.
\end{abstract}

Keywords: data hiding, FZDH etc.

\section{INTRODUCTION}

The World Wide Web have revolutionized the manner by which everything is accessible in computerized structure as it were. The wide spread and simple access to media content has roused advancement of innovations in computerized steganography or information stowing away with accentuation on get to control, confirmation, and copyright security. Security and protection assumes a fundamental job in information change. For secret communication, cryptography is a strategy that scrambles unique content or convert unique message into nonclear arrangement while steganography manages hiding a secret information in some transporter record which might be content, picture, sound and video record that can't be seen 
by unapproved individual. The video steganography utilizes an a few edges of video documents to implant a secret message and record.

The vast majority of the most recent work in information stowing away is about copyright insurance of media information. The upside of steganography over cryptography is that messages don't stand out to themselves. Information covering up in which a few information is passed on inside a host medium and transmitted to the recipient. There are four principle prerequisites of a run of the typical data hiding system:

Indistinctness: There ought not be perceptual debasement because of data hiding. In a perfect world one couldn't have the option to recognize have signal and checked sign. Checked sign ought to be like the host signal.

Robustness: It is the capacity and quality of an information concealing framework after specific attacks, as far as accurately interpreting the hidden information. The level of power is resolved by the application. All in all, information hiding algorithm is intended for specific attacks furthermore, permissible distortion levels.

Limit: It alludes to the practical number of message bits that can be covered up in the have signal. The sum may extend from one piece to a large number of bits, which relies upon the application.

Security: For certain applications security might be urgent. All things considered, algorithms should make sure about the hidden information with the goal that foes can't interrupt or interfere by any implies.

\section{DATA HIDING FRAMEWORK}

Computerized information data are spread and circulated everywhere throughout the world by utilizing web. Information covering up is the way toward embedding data into a host file. A structure of information hiding system is outlined in Fig. Right now, original digital media (I0 ), which is otherwise called the host media or spread media, the implanting module embeds in it a lot of secondary data (b ), which is alluded to as inserted information or watermark, to get the checked media (I1). The addition or inserting is done with the end goal that I1 is perceptually indistinguishable from I0 . The distinction between I1 and I0 is the twisting presented by the inserting procedure. By and large, the inserted information is an assortment of bits, which may originate from an encoded character string, from an example, or from some executable operators, contingent upon the application. The installed information (b) will be separated from the checked media (I1) by a finder, regularly after has experienced different preparing and assaults. The contribution to the locator is alluded to as test media (I2), and the separated information from I2 is signified byÙb. The distinction somewhere in the range of I 2 and I1 is called clamor. In such applications as proprietorship assurance, fingerprinting, and access control, precise unraveling of hidden information from mutilated test media is liked. The key components in numerous data hiding systems incorporate

- a perceptual model that guarantees imperceptibility; 
- a mechanism for embedding one bit;

- systems for implanting various bits through modulation / multiplexing; data

- how to deal with the pieces of host media in which it is hard to embeddata;

- how to upgrade robustness and security.

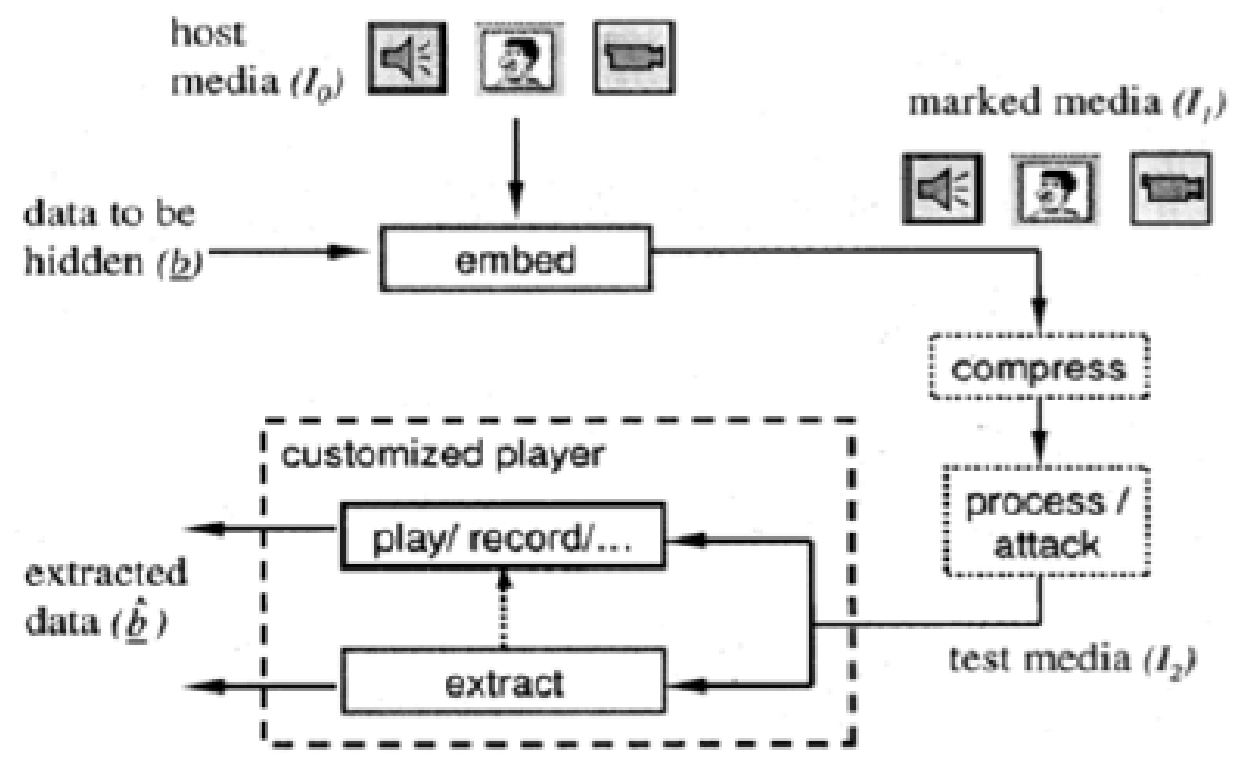

Fig1 General Framework of Data Hiding System

We can see these components through a layered structure appeared in Fig. The lower layers manage how one or various bits are implanted vaguely in the host media. Upper layers for accomplishing Equalization of lopsided limit, Error amendment, Security,Compression and encoding can be based over these lower layers.

\begin{tabular}{|l|c|}
\hline \multirow{2}{*}{ Upper Layers } & Compression and encoding \\
\cline { 2 - 2 } & Security \\
\cline { 2 - 2 } & Error Correction \\
\cline { 2 - 2 } & Equalization of uneven capacity \\
\hline \multirow{2}{*}{ Lower Layers } & Multiple - bit cmbedding \\
\cline { 2 - 2 } & Imperceptible embedding of one bit \\
\cline { 2 - 2 } &
\end{tabular}

Fig 2 Layered Structure of Data Hiding 


\section{FORBIDDEN ZONE DATA HIDING}

The proposed framework moving the protected information document utilizing video data hiding method that utilizes makes use of correction capacity of repeat accumulate codes andsuperiority of forbidden zone data hiding (FZDH). Fundamental inspiration of this proposed framework to hide and move the huge video record behind the littler size of spread document in secure way. The fundamental bit of leeway of utilizing video document hiding information from everything data is the included protection from of the outsider or unintended beneficiary because of the family member unpredictability of video contrasted with picture and sound document.

Forbidden Zone (FZ) is characterized as the host signal range, where modification isn't permitted during information hiding procedure. Forbidden Zone Data Hiding (FZDH) just utilizes FZ to modify the power imperceptibility exchange off. The meaning of FZ idea may prompt an impression of comparability among FZ and concealing, though they relate to entirely unexpected ideas.

Let $\mathrm{s}$ (striking indicating a vector) be the host signal in $\mathrm{RN}$ and $\mathrm{m} \in\{0,1\}$ be the information to be covered up. At that point the stamped signal $\mathrm{x}$ is acquired as yielded (1).

$$
x=\left\{\begin{array}{l}
s, s \in F Z m \\
M m(s), s \in A Z m
\end{array}\right.
$$

whereFZm, Allowed Zone (AZm) pair characterizes the host signal zones where change is permitted or not and $\mathrm{Mm}($.) is a mapping from $\mathrm{RN}$ to an appropriate segment of RN. The necessity on these zones and parcels is just founded on the limitation that they should be totally unrelated for various $\mathrm{m}$. The key purpose of FZDH is the assurance of the zones and the partitions.

There could be unbounded approaches to accomplish this; in any case, a functional plan can be performed by utilizing quantizers. Such a basic parametric structure is yielded (2), here the mapping capacity is characterized as:

$$
M m(s)=\{s+e m(1-r / e m)
$$

Here $\mathrm{r}$ is the control parameter, $\mathrm{Qm}($.$) is a quantizer ordered by \mathrm{m}$ and e is characterized as the distinction vector between the host sign and its quantized variant:

$$
-e m \triangleq Q-s
$$

The mapping capacity in (2) expresses that the host signal is changed by including an extra term, which is a scaled adaptation of the quantization contrast. In 1-D, this extra term is 
scalar, though in N-D have signal is moved along the quantization distinction vector and towards the recreation purpose of the quantizer. Consequently, installing contortion is decreased and decreased than the quantization error.

FZm and AZm are characterized utilizing the control parameter and the distinction vector:

$$
\begin{gathered}
F Z m=\{s \mid\|e m\| \leq r\}, \\
A Z m=\{s \mid\|e m\|>r\}
\end{gathered}
$$

Concealing is applied to data hiding and watermarking in various endeavors, as in request to fuse perceptual investigation, with the goal that perceptually usable host signal tests and reasonable contortion edges are resolved. Be that as it may, FZ doesn't include any perceptual investigation and versatile coefficient determination process. The primary inspiration of $\mathrm{FZ}$ is diminishing the implanting contortion at a specific translating mistake level. Like QIM, FZ ought to be applied, when the implanting bending is inside perceptually possible edges. This prerequisite is commonly fulfilled because of the have signal force limitation, which expresses that the host signal force is altogether more noteworthy than installing contortion. FZDH includes a set dividing to decide the scope of host signal where adjustment is permitted. FZDH utilizes a mapping in the AZ, or which quantizers are by all account not the only decision. In FZDH, at first all areas are taboo and one abatements these zones as per the ideal degree of unraveling blunder as for a channel clamor level. FZDH keeps a portion of the host signal unaltered. FZDH ways to deal with the information hiding issue from an alternate point of view than coding strategies: there exists uncoded segments of the host signal range. The fundamental inspiration is to keep the host signal unaltered for certain reaches, which ought to be decided by the ideal degree of robustness, embedding distortion amount and channel noise level.

\section{ADVANTAGES}

\section{Profoundly Secure}

Since arbitrary information are additionally set in unused frames in the video, the attacker is left dumbfounded to realize the genuine secret datahidden in the video. Henceforth exceptionally private information like military secrets and bank account can be effectively steganography in conventional video and can be transmitted over web even in unbound association.

\section{Limit}

Content based steganography has constrained limit and Image steganography attempted to improve the limit where half of original picture size can be utilized to conceal the mystery message. Be that as it may, there is impediment on how much data can be covered up into an picture. Video Steganography has been found to conquer this issue.

\section{Imperceptibility}


Least odds of detectable quality on account of rapidly showing of the frames, so it's become more enthusiastically to be suspected by human vision framework.

\section{APPLICATIONS}

\section{Secret Communication}

A distortion of arbitrary characters being transmitted between two clients may warn a attentive outsider that delicate data is being transmitted. The proposed method permits us to hide scrambled messages in mediums less inclined to draw in consideration.

\section{Copyright Protection}

A secret copyright notice or watermark can be inserted inside a picture/video to recognize it as licensed innovation. This is the watermarking situation where the message is the watermark. What's more, when a picture is sold or appropriated an distinguishing proof of the beneficiary and time stamp can be inserted to recognize potential privateers. A watermark can likewise serve to distinguish whether the picture has been in this way altered. Discovery of an implanted watermark is performed by a factual, correlation, or closeness test, or by estimating other amount trademark to the watermark in a stego-picture. The addition and examination of watermarks to secure copyrighted material is answerable for the ongoing flood of enthusiasm for computerized steganography and information inserting.

\section{Video Error Correction}

Since the transmission of any information is constantly dependent upon debasement because of mistakes, at that point the video transmission must arrangement with these mistakes without retransmission of undermined information. This is another application for steganography instead of security reason.

\section{Hiding the Military Secret Message}

Applications, for example, those of military, clinical and law authorization utilizes can't acknowledge indeed, even minor picture contortion. For instance significant mystery data, for example, the co-ordinates of foe's area can be covered up in a military guide for convert communication. In any case, an authority may settle on a wrong choice when a military guide is remade with distortion.

\section{Terrorist Attack}

Terrorists can likewise utilize steganography to keep their interchanges secret and to facilitate attacks. The entirety of this sounds genuinely evil, and in reality the conspicuous employments of steganography are for things like secret activities. In any case, there are various peaceful applications. The most straightforward and most seasoned are utilized in map making, where cartographers some of the time add a little anecdotal road to their maps, permitting them to indict copycats. A comparable stunt is to add anecdotal names to mailing records as a check against unapproved affiliates. 


\section{CONCLUSION}

Steganography is the specialty of hiding data and a push to hide the presence of the embedd data. It fills in as a superior method for making sure about message than cryptography which just disguises the substance of the message not the presence of the message. This work is exceptionally helpful to hide the all kind of data whilesending the significant and secret reports in hide video document; it will be imperceptible for unapproved individual.

\section{REFERENCE}

[1]. J. J. Chae , B. S. Manjunath," Data Hiding in Video", Department of Electrical and Computer Engineering University of California, Santa Barbara CA 93106-9560

[2]. Min Wu , Bede Liu ," Data Hiding in Image and Video: Part I-Fundamental Issues and Solutions" IEEE Transactions On Image Processing, Vol. 12 (6), 2003.

[3]. Arvind Kumar, Km. Pooja, "Steganography- A Data Hiding Technique" International Journal of Computer Application, Vol. 9, No.7, November 2010.

[4]. T. Morkel, J.H.P. Eloff, M.S. Olivier, “An Overview of Image Steganography” Information and Computer Security Architecture (ICSA) Research Group Department of Computer Science University of Pretoria, 0002, Pretoria, South Africa .

[5]. Hafiz Malik , K. P. Subbalakshmi ,R. Chandramouli ," Nonparametric Steganalysis of QIM Steganography using Approximate Entropy”, Electrical and Computer Engineering Department, 